\title{
Multidisciplinary management of very advanced stage III and IV melanoma: Proof-of-principle
}

\author{
HAIM GUTMAN $^{1}$, EYTAN BEN-AMI ${ }^{2}$, RONI SHAPIRA-FROMMER $^{2}$ and JACOB SCHACHTER ${ }^{2}$ \\ ${ }^{1}$ Department of Surgery, Rabin Medical Center, Beilinson Campus; \\ ${ }^{2}$ Ella Institute for Treatment and Research of Melanoma, \\ Ch Sheba Medical Center, Tel Aviv University, Sackler School of Medicine, Israel
}

Received January 7, 2012; Accepted March 19, 2012

DOI: $10.3892 / \mathrm{ol} .2012 .712$

\begin{abstract}
Patients with potentially resectable advanced stage III and IV melanoma are a selected subgroup that gain maximal advantage if treated in a melanoma center. Surgery combined with chemo/chemobiotherapy may yield durable remission and long-term palliation. Thirty-seven non-randomly selected patients underwent systemic therapy with the aim of consolidating treatment by surgery. Data were collected prospectively, and analyzed retrospectively. The median follow-up from diagnosis was 50 (3-307) months and 15 (1-156) months when calculated from the last intervention. Twenty-two males and 15 females, with a median age at diagnosis of 44 (20-71) years, with 13 trunk, 13 extremity, 3 head and neck and 8 unknown primary melanomas were included. There were 17 stage III and 20 stage IV patients with a median Breslow thickness of 3.7 (0.45-26) mm. Chemo/ chemobiotherapy achieved 7 clinical complete responses (cCRs), 28 partial responses (PRs) and 2 instances of stable disease. Six of the 7 cCRs were operated on, securing pathological complete response in 5 and PR in one. Four of these five and the PR patient still have no evidence of disease (NED). Twenty-one of 30 PR patients were rendered NED by surgery; 14 of these 21 patients succumbed to melanoma, and one is alive with stable disease. Overall, 11 of 37 patients have not succumbed to melanoma, with a median of 72 (14-156) months survival following the last intervention. Of the eight patients with unknown primary melanomas, five have not succumbed to melanoma, with a median of 89 (30-156) months survival following the last intervention. Patients with marginally resectable stage III and IV melanoma have a significant $30 \%$ chance, according to this series, for durable remission if treated by a multidisciplinary team in a melanoma center using induction
\end{abstract}

Correspondence to: Dr Haim Gutman, Department of Surgery, Rabin Medical Center, Beilinson Campus, Petach Tikva 49100, Israel

E-mail: hgutman@post.tau.ac.il

Key words: melanoma, loco-regionally advanced, chemobiotherapy, neoadjuvant, multidisciplinary, sustained remission, complete response, surgery, metastatic chemobiotherapy and surgery. Results are more favorable for patients with an unknown primary lesion. In view of the currently approved new effective treatments for melanoma, this study may be considered a proof-of-principle investigation, enabling long-term remissions by combining induction therapy and surgery.

\section{Introduction}

Based on the 2006 Surveillance Epidemiology and End Results (SEER) database, the incidence of melanoma is 19.6 per 100,000 population per year in the US, with mortality rates at 2.7 per 100,000 (1). Rates in Israel are not significantly different with 1,200 new cases being reported per year per 7,000,000 population. The favorable incidence to mortality ratio reflects the fact that most melanoma patients are diagnosed at an early, surgically curable stage. Approximately 8,400 patients per year in the US either presented with or developed incurable disease, and succumbed to melanoma (1).

Although surgery provides the best opportunity for cure, it is frequently futile or impossible in patients with advanced stage III or IV metastatic disease. Thus, a multidisciplinary approach is an option that should be applied to this subgroup of patients, in an attempt at improving their outcome.

In their 1998 phase II chemobiotherapy (CBT) study, Buzaid et al achieved $6.5 \%$ pathological complete response $(\mathrm{pCR})$ and $43.5 \%$ partial response (PR) in 65 potentially resectable stage III melanoma patients (2). Stein et al used CBT to achieve $17 \%$ CR in 29 stage IV patients with 8 months median time to progression (3). Gibbs et al reported clinical complete response (cCR) in $2.8 \%$ of 48 stage III patients, pCR in $11.1 \%$ and $36 \% \mathrm{PR}$, using combined preoperative and postoperative CBT, with $64.6 \%$ of patients being disease-free following a median follow-up of 31 months (4). Researchers from Yale University identified 16 patients who responded to combination chemotherapy and had their residual tumor resected. Eleven of these 16 were alive with no evidence of disease (NED) following a median of 35 months (5).

Our own experience with CBT echoes the results of these publications with a $44 \%$ (28 of 57 patients) response rate, $25 \%$ (14 patients) CR and 19\% (11 patients) PR (6).

Based on these observations, and specifically, the high response rate, the CBT regimen was considered and applied 
as the treatment of choice to precede or be combined with surgery for selected patients who were deemed potentially resectable or became potentially resectable during treatment.

\section{Patients and methods}

Patient selection. The Institutional Melanoma Tumor Boards selected 37 cutaneous melanoma patients diagnosed between January 1983 and October 2006 in a non-randomized manner. These patients presented with or developed very advanced stage III or IV disease, and were deemed eligible for induction CBT followed by potentially curative surgery (Table I).

Very advanced stage III was defined as irresectable or marginally resectable regional disease, based on surgical oncologist's clinical examination, computed tomography (CT) or positron emission tomography-computed tomography (PET-CT) plus magnetic resonance imaging (MRI) of brain and multidisciplinary team deliberation. Stage IV for inclusion in this study required biopsy-proven distant metastases combined with progression on imaging.

The study was approved by the ethics committee of the hospital. Signed informed consent was obtained from the patient prior to commencing chemotherapy treatment.

Methods. Patients had to be on no active treatment for at least two weeks prior to the initiation of CBT. All 37 patients had an ECOG performance status of $0-2$; white blood cell count $>3000$; platelet count $>100,000$; normal renal function tests; bilirubin $<2 \mathrm{mg} \%$; and normal thyroid function tests. Symptomatic patients or those aged $>50$ underwent a Thallium 201 Effort Stress Cardiac Perfusion Test and/or lung function test $(>75 \%$ of predicted). Systemic evaluation included head, chest and abdominal CT or PET-CT with MRI of brain. Patients on corticosteroids, with other malignancies or brain metastases or known mucosal primary malignancies were excluded.

Two chemotherapy regimens were used (Table II), both using interferon- $\alpha$ (Intron $\mathrm{A}^{\circledR}$; Schering, Kenilworth, $\mathrm{NJ}$, USA), dacarbazine and cisplatin. BCNU and granulocytemonocyte colony stimulating factor (GMCSF), which were part of the first regimen were replaced by decrescendo interleukin 2 (IL-2) (Proleukin ${ }^{\circledR}$; Hoffman La Roche, Nutley, NJ, USA) due to the questionable contribution of BCNU and its significant side-effects, the unavailability of GMCSF in Israel, and the activity of IL-2 in metastatic melanoma.

Response evaluation was performed at the end of every two courses combining clinical examination, CT or PET-CT comparative study and multidisciplinary team evaluation. Surgical intervention was considered at each interval. CBT was stopped if progressive disease was noted.

\section{Results}

Thirty-seven patients were included in this analysis (Table I, Fig. 1), of whom 4 did not undergo surgery. Of these 4 patients, 2 patients never became resectable, and succumbed to their disease. One patient achieved temporary CR (PET-CT), but had a recurrence prior to consenting to undergo surgery. The fourth patient is alive with NED 108 months following emergency surgical removal of large metastatic bleeding ulcerating axillary disease of unknown primary malignancy and the
Table I. Patient characteristics.

\begin{tabular}{lr}
\hline Total number of patients & 37 \\
Male/female & $22 / 15$ \\
Age at diagnosis (years) & \\
Median/range & \\
Melanoma site & 14 \\
Trunk & 13 \\
Extremity & 3 \\
Head and neck & 8 \\
Unknown primary & \\
Breslow thickness (mm) & \\
Median/range & $17 / 20$ \\
Stage & 13 \\
Advanced III/IV & \\
Earlier CBT regimen & \\
Number of patients & \\
Median number of courses (range) & $24-6$ (2-6) \\
Later CBT regimen & \\
Number of patients & $4(2-6)$ \\
Median number of courses (range) & \\
\hline
\end{tabular}

a See Table II; CBT, chemobiotherapy.

disappearance of biopsy proven liver and prostate metastases. Thirty-four patients underwent surgery following a median of 4 CBT courses, and 27 achieved CR. Patients who did not have surgery $(\mathrm{N}=3)$, and those in whom surgery failed to remove all residual disease $(\mathrm{N}=7)$, succumbed to melanoma. Similarly, 16 patients who were rendered disease-free by CBT and surgery combined succumbed to the disease.

Of the 37 patients, 11 patients (30\%) did not succumb to melanoma during the median follow-up period of 72 (14-156) months following the last intervention. One of these 11 patients succumbed to non-small-cell lung cancer 87 months following the last intervention for melanoma. Another patient was alive with diminishing disease and expanding vitiligo 25 months from the last intervention, at the time of data analysis. The patient remains alive with no active disease over 30 months later.

Five of 8 patients with unknown primary survived with NED for a median of 89 (30-156) months since the last intervention.

\section{Discussion}

CBT regimens have been employed to treat melanoma patients. Although high response rates were documented $(2-4,6)$, phase III studies failed to show survival advantage to CBT over the standard single agent DTIC, and were associated with significant toxicity (7). Furthermore, the exact role of each component of these multi-agent regimens has not been well defined. Thus, these regimens have not matured into a standard first-line treatment. A number of clinical studies are ongoing to find the best regimen. By applying modern targeted 


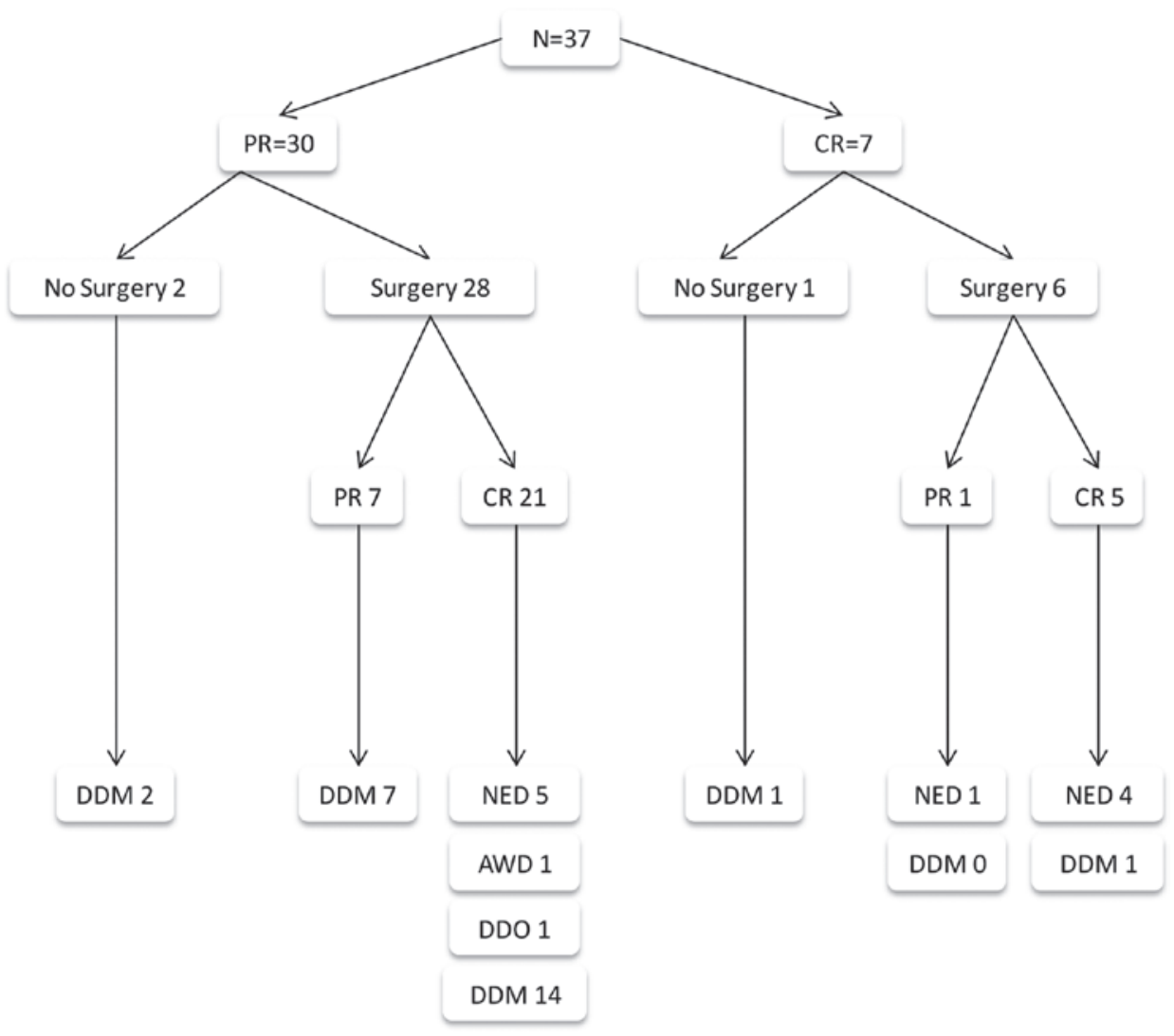

Figure 1. Number of patients undergoing multidisciplinary chemobiotherapy and surgery with treatment outcome. PR, partial response; $\mathrm{CR}$, complete response; DDM, died due to melanoma; NED, no evidence of disease; AWD, alive with disease; DDO, dead due to other cause.

Table II. Chemobiotherapy (CBT) regimens.

A, Earlier regimen (N=13), Q-21 day (16).

\begin{tabular}{lrr}
\hline Regimen & Dose & Initiation point \\
\hline INF $\alpha$ (s.c.) & $3 \mathrm{mU} /$ days $1,3,5$ & Week I \\
DTIC (i.v.) & $220 \mathrm{mg} / \mathrm{m}^{2} /$ days $1-3$ & Week II \\
CDDP (i.v.) & $25 \mathrm{mg} / \mathrm{m}^{2} /$ days $1-3$ & Week II \\
BCNU (i.v.) & $150 \mathrm{mg} / \mathrm{m}^{2} /$ day 1 & Week II \\
& & (every 2nd course) \\
GMCSF (s.c.) & $20 \mu \mathrm{g} / \mathrm{m}^{2} /$ days 1-7 & Week III \\
\hline
\end{tabular}

B, Later regimen $(\mathrm{N}=24), \mathrm{Q}-21$ day.

\begin{tabular}{lc}
\hline Regimen & Dose \\
\hline INF $\alpha$ (s.c.) & $5 \mathrm{mU} /$ days $1-21$ \\
DTIC (i.v.) & $220 \mathrm{mg} / \mathrm{m}^{2} /$ days $1-3$ \\
CDDP (i.v.) & $25 \mathrm{mg} / \mathrm{m}^{2} /$ days $1-3$ \\
IL-2 (i.v.) & $18 \mathrm{mU} / \mathrm{m}^{2} /$ day 1 \\
& $9 \mathrm{mU} / \mathrm{m}^{2} /$ day 2 \\
& $4.5 \mathrm{mU} / \mathrm{m}^{2} /$ days $3-4$
\end{tabular}

s.c., subcutaneous; i.v., intravenous; INF, interferon $\alpha$ 2b; DTIC, dacarbazine; CDDP, cisplatin; IL-2, interleukin 2; GMCSF, granulocyte-monocyte colony stimulating factor. approaches, two of these new studies yielded two new highly effective, life-prolonging, drugs in metastatic melanoma.

The high response rates of CBT regimens have also been the focus of other studies. Hwu et al rendered 3 of 35 stage IV patients NED by surgery following temozolomide and interferon induction treatment (8). In their study, Lewis et al reported a $26 \%$ response rate using preoperative and postoperative CBT (9). Even peri-operative interferon alone was able to achieve up to $55 \%$ PR with $15 \%$ pCR (10). However, a more recent combination containing cisplatin, paclitaxel and sorafenib failed to induce a response rate of this magnitude (11).

The traditional CBT regimens created the best opportunity for a significant response during the study period (7). Thus, it seemed reasonable to regard these patients as resectable and attempt surgery to render the responders NED in order to secure long-term remission.

The role of surgery is not well defined in those patients rendered cCR by the induction therapy alone. The mere presence of microscopic residual disease places the patients at a potential risk of recurrence, thus, surgery appears crucial to secure CR and long-term remission. The present study may serve as a proof-of-principle, since $30 \%$ long term remission achieved is not perceived as typical natural history of advanced stage III and IV melanoma.

The use of a priori CBT is significant for various reasons. Quality of life indices are better in responders compared to supportive care, and responders are more likely to benefit from further treatments (12), as demonstrated in the present 
series. Surgery for responders may be of a lesser extent and less mutilating. Surgery may be omitted for non-responders. Hypothetically, the induction therapy also 'sterilizes' the periphery of the tumor mass, thus reducing the surgical field and intravascular tumor cell shedding (13). Although direct evidence to support this hypothesis is scarce, the overall results of this study may be, in part, due to such an effect. Response to CBT facilitates and encourages surgical attempts. Disease progression is an indicator that CBT treatment is more beneficial than surgery. Surgery for responders results in clinically meaningful prolongation of remission in an, admittedly low, but significant percentage of these advanced stage patients.

Numerous studies in favor of surgical resection of metastatic melanoma in selected populations have been published and reviewed $(14,15)$. Adding induction CBT may improve patient selection for surgery, reduce its extent, and improve quality of life. Patients with reasonable performance status, whose metastatic disease may become resectable, should be offered the option of this multidisciplinary approach, which is currently the only one with up to $30 \%$ chance of an unmaintained remission.

Recently, extensive clinical experience has been gained with the use of new technologies for advanced melanoma such as B-RAF inhibitors and anti CTLA-4 antibodies. The group of the B-RAF inhibitors has shown a notable, albeit short clinical response of more than $50 \%$ and is potentially applicable to the above neoadjuvant multidisciplinary approach (Chapman et al, American Society of Clinical Oncology 2011 meeting, abs. LBA4, 2011).

\section{References}

1. National Cancer Institute: SEER Cancer Statistics Review, 1975-2006. http://seer.cancer.gov/csr/1975_2006/. Accessed: April 12, 2012.

2. Buzaid AC, Colome M, Bedikian A, Eton O, Legha SS Papadopoulos N, Plager C, Ross M, Lee JE, Mansfield P, et al: Phase II study of neoadjuvant concurrent biochemotherapy in melanoma patients with local-regional metastases. Melanoma Res 8: 549-556, 1998.

3. Stein ME, Bernstein Z, Drumea K, Zalik M, Shklar Z, Steiner M and Haim N: Sustained complete remission after chemobiohormonal therapy for metastatic melanoma. Am J Clin Oncol 22: 62-64, 1999.
4. Gibbs P, Anderson C, Pearlman N, LaClaire S, Becker M, Gatlin K, O'Driscoll M, Stephens J and Gonzalez R: A phase II study of neoadjuvant biochemotherapy for stage III melanoma. Cancer 94: 470-476, 2002.

5. Sasson HN, Poo WJ, Bakas MH and Ariyan S: Prolonged survival in patients with advanced melanoma treated with neoadjuvant chemotherapy followed by resection. Ann Plast Surg 37: 286-292, 1996.

6. Bar J, Yerushalmi R, Shapira-Frummer R, Kutchuk I, Sulkes A,Gutman H, Catane R and Schachter J: Concurrent chemobiotherapy with cisplatin, dacarbazine, decrescendo interleukin-2 and interferon $\alpha 2 b$ in patients with metastatic melanoma. Oncol Rep 20: 1533-1538, 2008.

7. O'Day S and Boasberg P: Management of metastatic melanoma 2005. Surg Oncol Clin N Am 15: 419-437, 2006.

8. Hwu WJ, Panageas KS, Menell JH, Lamb LA, Aird S, Krown SE, Williams LJ, Chapman PB, Livingston PO, Wolchok JD and Houghton AN: Phase II study of temozolomide plus pegylated interferon-alpha-2b for metastatic melanoma. Cancer 106: 2445-2451, 2006.

9. Lewis KD, Robinson WA, McCarter M, Pearlman N, O'Day SJ, Anderson C, Amatruda TT, Baron A, Zeng C, Becker M, et al: Phase II multicenter study of neoadjuvant biochemotherapy for patients with stage III malignant melanoma. J Clin Oncol 24: 3157-3163, 2006

10. Moschos SJ, Edington HD, Land SR, Rao UN, Jukic D, Shipe-Spotloe J and Kirkwood JM: Neoadjuvant treatment of regional stage IIIB melanoma with high-dose interferon alfa-2b induces objective tumor regression in association with modulation of tumor infiltrating host cellular immune responses. J Clin Oncol 24: 3164-3171, 2006.

11. Hauschild A, Agarwala SS, Trefzer U, Hogg D, Robert C, Hersey P, Eggermont A, Grabbe S, Gonzalez R, Gille J, et al: Results of a phase III, randomized, placebo-controlled study of sorafenib in combination with carboplatin and paclitaxel as second-line treatment in patients with unresectable stage III or stage IV melanoma. J Clin Oncol 27: 2823-2830, 2009.

12. Sarnaik AA, Zager JS and Sondak VK: Multidisciplinary management of special melanoma situations: oligometastatic disease and bulky nodal sites. Curr Oncol Rep 9: 417-427, 2007.

13. Stephens FO: Induction chemotherapy: to downgrade aggressive cancers to improve curability by surgery and/or radiotherapy. Eur J Surg Oncol 27: 672-688, 2001.

14. Gutman H, Hess KR, Kokotsakis JA, Ross MI, Guinee VF and Balch CM: Surgery for abdominal metastases of cutaneous melanoma. World J Surg 25: 750-758, 2001.

15. Mosca PJ, Teicher E, Nair SP and Pockaj BA: Can surgeons improve survival in stage IV melanoma? J Surg Oncol 97: 462-468, 2008.

16. Schachter J, Rakowsky E, Sulkes A and Adler A: A sequential four-drug chemotherapy and biotherapy with interferon alpha and GM-CSF - an innovative protocol for the treatment of metastatic melanoma. Cancer Biother Radiopharm 13: 155-164, 1998. 